Methods This was a single-centre clinical outcome study of pathological variables, including elastica detected venous invasion, in stage I-III electively resected colorectal cancer specimens.

Results 631 resection specimens, excised between 1997-2009, were analysed (176 retrospectivley and 455 prospectively). The median follow up was 73 months (24-178) and during this time there were 238 deaths (134 from cancer). Venous invasion was detected in $56 \%$ of cases and was a stronger predictor of poor long term cancer-specific survival than other pathological features on univariate and multivariate analyses. On multivariate analysis of all cases the hazard ratio (HR) for failure to survive 5 years for venous invasion $=3.94$ (95\%CI 2.33-6.65, P < 0.001); HR for lymph node involvement $=1.81,(95 \%$ CI 1.43-2.30, $\mathrm{P}<0.001)$ and $\mathrm{HR}$ for $\mathrm{T}$ stage $=1.64(95 \% \mathrm{CI} 1.16-2.30, \mathrm{P}=0.005)$. In node negative cases the HR for failure to survive 5 years for venous invasion on multivariate analysis $=3.55(95 \% \mathrm{CI} 1.81-6.97, \mathrm{P}<0.001)$ and for $\mathrm{T}$ stage was $2.03(95 \%$ CI $1.26-3.28, \mathrm{P}=0.004)$. Venous invasion strongly related to other high-risk pathological variables. In cases with no venous invasion, no pathological characteristic related to survival other than $\mathrm{T}$ stage. When $\mathrm{T}$ stage and venous invasion were considered together, patients could be stratified by risk of 5 -year cancer mortality from $100-54 \%$ in node negative disease and $100-33 \%$ in node positive disease.

The importance of elastica detected venous invasion can be appreciated from the development of a novel staging system based only on T stage and venous invasion (TVI). This simple TVI system was at least as predictive as the gold standard TNM system when considering all cases, and provided increased prognostic value in both T1 and T2 tumours, as well as in node negative disease.

Conclusion Sensitive, accurate detection of venous invasion on elastica stained sections improves its prognostic importance such that it becomes a key pathological characteristic, arguably of more importance than nodal status, in determining outcome in patients with colorectal cancer. TVI staging provides a novel and simple method by which venous invasion coupled with T stage can be utilised to predict survival.

Disclosure of Interest None Declared

\section{PWE-005 IS INVESTIGATION OF THE COLONIC LUMEN NECESSARY AFTER CT DIAGNOSIS OF ACUTE DIVERTICULITIS?}

doi:10.1136/gutjnl-2013-304907.294

1."A Tan, ' $\mathrm{C}$ Newman. ' Colorectal Surgery, Airedale NHS Foundation Trust, Keighley, UK

Introduction Traditionally, it is recommended that the colon be evaluated after an episode of acute diverticulitis to exclude underlying pathologies such as malignancy. However, the necessity of this has been questioned in this era of frequent use of contrast-enhanced multidetector computed tomography (CT), which itself is a moderately sensitive test for colorectal cancer, to diagnose acute diverticulitis. This study aimed to determine the yield of malignancy from subsequent follow-up investigations or treatments in patients after an episode of CT-proven acute diverticulitis.

Methods This was a retrospective study of patients with acute diverticulitis as diagnosed on CT at a moderately-sized district general hospital between January 2007 and December 2011. Uncomplicated diverticulitis was defined as the presence of colonic diverticular disease with localised colonic wall thickening and/or inflammation of pericolic fat. Complicated diverticulitis was defined as the additional presence of abscess, obstruction, fistula or evidence of perforation.

Results A total of 64 patients were diagnosed with acute diverticulitis on contrast-enhanced CT. Thirty-five patients had uncomplicated diverticulitis and 29 patients had complicated diverticulitis.

The follow-up of patients with uncomplicated disease were as follows: flexible sigmoidoscopy (10), colonoscopy (4), CT colonography
(2) and barium enema (4). Two patients underwent surgery. All investigations or treatments confirmed diverticular disease. None of them showed underlying malignancy. Incidental findings at the diseased sites were detected in 4 patients. Three of them had polyps and one had an aphthous ulcer. Of the patients with polyps, one had a tubular adenoma greater than $10 \mathrm{~mm}$, one had a $2 \mathrm{~mm}$ tubular adenoma with low-grade dysplasia and one had an inflammatory polyp. The biopsy of the patient with the ulcer was normal. The remaining 13 patients had no follow-up and none of them were known to have returned with malignancy.

The follow-up of patients with complicated disease were as follows: flexible sigmoidoscopy (9), colonoscopy (4), CT colonography (1) and barium enema (5). Six patients had surgery. All investigations or treatments confirmed diverticular disease with no evidence of underlying malignancy. A patient who underwent surgery incidentally had hyperplastic polyps in the sigmoid colon specimen. Four patients had no follow-up and none of them were known to have developed malignancy.

Conclusion The yield of malignancy as an underlying pathology was nil in both cohorts. Routine investigation of the colonic lumen after an episode of acute diverticulitis in this era of widespread CT use may not be necessary in the absence of other sinister indications. Limited resources may be better utilised for other patients with greater risk of colorectal cancer.

Disclosure of Interest None Declared

\section{PWE-006 HOW OFTEN IS BOWEL CANCER DETECTED FROM A POSITIVE 3RD KIT IN THE ENGLISH BCSP?}

doi:10.1136/gutjnl-2013-304907.295

1."A Field, 'M Vogler, ${ }^{1} \mathrm{R}$ F Logan. 'Eastern Bowel Cancer Screening Hub, Nottingham University Hospitals Trust, Nottingham, UK

Introduction In the English Bowel Cancer Screening Programme (BCSP) subjects returning a weak positive kit (1-4 of the 6 windows positive) are invited to do a $2^{\text {nd }}$ kit and if none of the windows are positive they are invited to complete a $3^{\text {rd }}$ kit. If any windows are then positive subjects are referred for possible investigation; if no windows are positive subjects are discharged from that screening round. This testing algorithm has been criticised for making the screening process too prolonged thereby producing anxiety and drop-outs and the Scottish BCSP has abandoned asking for a $3^{\text {rd }}$ kit on the grounds that the yield was negligible.

Methods We have analysed the outcomes from the $3^{\text {rd }}$ kits returned to the Eastern BCSP Hub from subjects invited for screening between 1 Jan 2011 and 31 March 2012.

Results Over this period over 850,000 subjects aged 60-74 yrs were invited for screening. $4 \%(20,021)$ completed 3 kits and of these $16 \%$ (3192) had a positive $3^{\text {rd }}$ kit and were referred for further investigation. Of those investigated (2830) 4.4\% (125) were found to have bowel cancer compared with $17.8 \%$ (298) with cancer found after a single kit and $8.1 \%$ (483) with cancer found after completing 2 kits. A further $7 \%$ and $12 \%$ completing 3 kits were found to have high and intermediate risk adenomas. The mean time from selection for screening to obtaining a definitive result for those completing 3 kits was 63 days compared to 34 days for those completing a single kit ( $95 \%$ of all subjects returning kits) and 49 days for those completing 2 kits ( $1 \%$ of all subjects returning kits).

Conclusion A significant number (14\%,125/906) of bowel cancers are detected in those completing 3 kits but this is at the cost of having a screening episode prolonged to almost twice that for subjects obtaining a definitive result after one kit. The intended introduction of faecal immunochemical tests to replace guaiac faecal occult blood tests should allow the use of a simpler and shorter testing algorithm

Disclosure of Interest None Declared. 